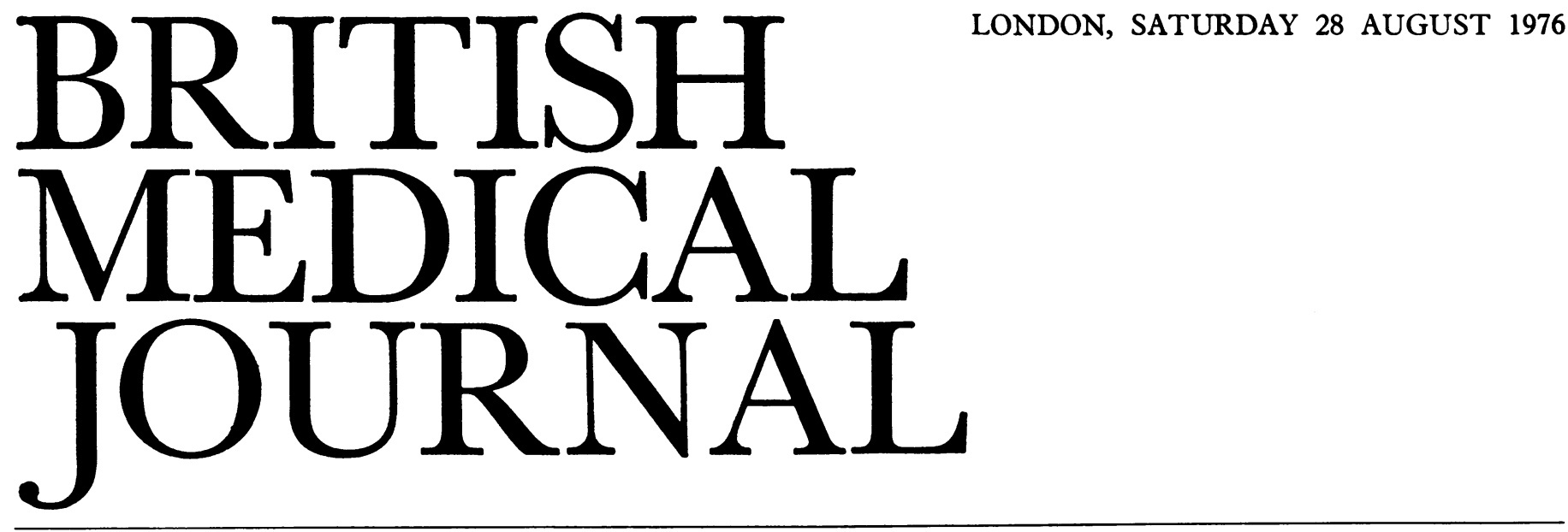

\title{
Perforated duodenal ulcer
}

In 1894 Hcnry Percy Dean ${ }^{1}$ of the London Hospital reported the first successful operation in a hitherto fatal conditionperforation of a duodenal ulcer. His patient was a woman of 27 with a 24-hour history of acute abdominal pain. The edges of the perforation were sutured, the peritoneal cavity washed out, and the wound closed without drainage. Two months later the unfortunate woman died of intestinal obstruction due to a band, but necrospy showed no sign of the previous ulcer.

While the incidence of perforation appears to be declining from its highest peak at the end of the second world war, it is still a common surgical emergency. Mackay and Mackay ${ }^{2}$ found that in Glasgow there was a 4.4 to 1 male to female ratio and there were nearly nine times as many duodenal as gastric perforations. The peak incidence was at New Year, and men tended to perforate on Fridays and Saturdays-after the factories had closed and the public houses had opened. The overall mortality was $11 \%$, and (as in previous studies) it increased with the age of the patient and the delay in surgical intervention. Provided the patient is otherwise fit, the diagnosis (which is seldom difficult) is made promptly, and surgery is started at once, the immediate outlook for survival today is good. But what is the ultimate prognosis of a patient who has his perforation repaired, and what is the emergency operation of choice?

Until recent years simple closure of the perforation was almost universal. Gastrectomy was used in those relatively rare cases where there was recurrence of a perforation, associated stenosis, or, most pressingly, concomitant haemorrhage. Simple closure of the perforation is insufficient to stop bleeding, which often comes from the posterior aspect of an ulcer straddling much of the circumference of the duodenum.

The outlook after simple repair depends largely on whether the perforation is an acute episode affecting a previously healthy duodenum (when the outlook is relatively good) or part of the progressive natural history of a chronic peptic ulcer (when recurrent symptoms are very likely). Cassell, ${ }^{3}$ for example, found that only $20 \%$ of patients having suture of an acute perforation with a history of less than three months required subsequent gastrectomy compared with $43.5 \%$ of those with chronic ulcers, and other series have put the relapse rate as high as $85 \%$ with chronic ulcers. Griffin and Organ ${ }^{4}$ did a long-term follow-up of 76 patients who had undergone simple repair and found that five died of later complications of their ulcer (one perforation and four haemorrhages), and that 39 required reoperation-34 elective and five emergency operations for a second perforation. Recurrence of symptoms, reoperation, and higher mortality were characteristic of the $80 \%$ of cases who had a long previous history of duodenal ulceration.

Because of this poor long-term prognosis more and more surgeons have been turning to more radical surgery at the time of the emergency procedure. Originally the operation chosen was emergency partial gastrectomy, but more recently the choice has switched to vagotomy and drainage with excision of the perforated ulcer in the fashioning of the pyloroplasty. Early fears of a danger of a mediastinitis as a result of opening the oesophageal hiatus in the presence of peritoneal contamination have proved unfounded. Hinshaw and his colleagues first started performing vagotomy and pyloroplasty for perforation in 1955 and reported 180 examples with only two deaths $(1 \cdot 1 \%){ }^{5}$ They went on to advise this procedure in an increasingly wide spectrum of cases. Hadfield and Watkin ${ }^{6}$ reported three deaths in 61 cases $(5 \%)$, selected because of their long history, previous perforation, or the chronicity of the ulcer found at operation.

Apparently there have been no randomised prospective trials comparing the two techniques. Nor, indeed, would these be justified, since even those surgeons who advise radical surgery avoid so doing in most poor-risk late cases with established peritonitis or even abscess formation. Thus Jordan and Korompai, ${ }^{7}$ who in a randomised prospective survey compared vagotomy and drainage with vagotomy and hemigastrectomy in perforation, rejected 40 out of 157 patients for the trial because of these conditions and treated them with simple closure. Perhaps not surprisingly there were no fewer than eight early deaths in these seriously ill patients $(20 \%)$, compared with only one postoperative death in the 117 patients undergoing definitive surgery. There was little to choose between the two types of curative operation, and the longterm results were about the same as those that might have been expected from elective surgery. Jordan and Korompai have now performed 13 simple closures combined with a highly selective vagotomy (confined to denervation of the parietal cell mass) in pat ents who were candidates for definitive treatment because of the chronicity of their ulcers. There were no deaths, no serious morbidity (apart from one intestinal obstruction due to adhesions), and the results were satisfactory in a short follow-up of 21 months. If these results are maintained in the long term this may indeed provide the operation of choice for the uncomplicated but chronic perforated duodenal ulcer.

What, then, is the wisest course of treatment? In Britain 
much emergency surgery is carried out by junior staff, at night, and in less than ideal conditions, and we must expect that circumstances may deteriorate still further. Under these adverse conditions the simplest possible life-saving procedure should be carried out-repair of the perforation using an omental patch. This also remains the wisest move in the desperately ill or elderly patient or one in whom the perforation has been present for 24 hours or more and in whom there is established peritoneal sepsis. Once life has been saved the patient may be carefully reviewed and definitive surgery subsequently advised if he has an undoubted chronic duodenal ulcer or if, in a previously asymptomatic patient, symptoms subsequently appear. In expert hands, and in good conditions, further hospital admission can be avoided in the patient with a chronic ulcer who is otherwise fit by performing definitive surgery at the time of perforation; the mortality should be low and the functional result good. Most surgeons would now perform a vagotomy and pyloroplasty in these circumstances except in the face of active haemorrhage-a relatively rare but lethal combination-in which case a gastrectomy of the Polya type is the operation of choice. Possibly the best combination may prove to be highly selective vagotomy combined with simple repair of the perforation. Nevertheless, we must wait for the long-term evaluation of the results of this relatively untried procedure.

\footnotetext{
${ }^{1}$ Dean, H P, Lancet, 1894, 1, 1191.

2 Mackay, C, and Mackay, H P, British fournal of Surgery, 1976, 63, 157.

3 Cassell, P, Gut, 1969, 10, 572.

4 Griffin, G E, and Organ, C H, Annals of Surgery, 1976, 183, 382.

${ }^{5}$ Hinshaw, D B, et al, American fournal of Surgery, 1968, 115, 173.

${ }^{6}$ Hadfield, J I, and Watkin, D F L, British Medical fournal, 1964, 2, 12.

7 Jordan, P H, and Korompai, F L, Surgery, Gynecology and Obstetrics, $1976,142,391$
}

\section{Patients' days}

The committee of the Central Health Services Council which was asked "to consider and advise on the principles which should govern the organisation of the inpatient's day in hospitals other than psychiatric hospitals" took five years to produce its report. ${ }^{1}$ Far more than a timetable, taking into account not only the mechanics of living and dying in hospital but all the circumstances and pressures that influence them, it is full, sensible, and readable. But many of the problems discussed, such as early waking, noise, and difficulties over mealtimes, have been with us for many years, and the lack of apparent progress is disappointing.

The priorities for most hospital patients would surely be the services of a technically skilled staff trained in human relations and communications together with adequate hotel resources. A carpet on the floor and a choice of menus would come further down the list. The difficulty about improving the quality and amount of information that patients receive is that the medical and nursing professions are hierarchical in structure, given to caution (for a variety of reasons) in imparting facts or forecasts, and notoriously better trained in professional techniques than in the attitudes and skills of communication. "The patient comes first" is an admirable sentiment, but hardly a guide in the complexities of relations in a ward. "The dignity of the individual" is the phrase in current use, but this too will remain a cliche unless maintained by the ability to perceive the patient's needs and exerting the will to meet them. Mr David Ennals has endorsed this report, and he too puts improvement in providing information for patients at the top of his list of desirable developments. Changing staff attitudes and teaching them to talk more and more freely to patients need not cost the NHS a lot of money.

His second point is also one that costs nothing: visiting hours should be flexible in all hospitals. Merely lengthening them is not the best solution, for relatives feel obliged to stay for the whole period, and both they and the patient are fatigued. It is especially important in paediatric wards, where accommodation for a mother (or father) to stay with a sick child is absolutely necessary, and the report also suggests that units should be available for families if the mother has other children she cannot leave. The consultant's role in a paediatric ward, apart from his rounds, is said to be to show "ready availability for consultation with residents and parents at any time in the 24 hours. He should set an example of ward behaviour to children, parents, and residents, and support them in serious and especially in fatal illness. He should encourage all the ward staff to contribute to ward policy and do his best to help the younger doctors and nurses to talk naturally to all ages of children and all sorts of parents."

Among avoidable nuisances those that cause noise rank high-radio and television, telephone bells, cleaning equipment, public address systems, and thoughtless staff. Admissions to wards at night, poor temperature control, and the nuisance from smoking are among the other ways in which the quality of hospital life can be lowered. Some factors are difficult to alter, though they affect standards of care. These include shift systems; public transport problems for staff at unsocial hours; lack of domestic and catering staff in the evenings, which may cause undesirably early supper times; and staff shortages of all kinds. Old buildings which reflect in their sanitary arrangements the past days, in which most patients stayed in bed all day, are poorly provided with lavatories, bathrooms, and showers.

Dying is a part of living, and there is a chapter on its management in hospitals. All nurses and medical students should be taught the proper care of the terminally ill, and the aim must be that the end should be free of suffering, peaceful, and dignified. Patients' welfare is, however, bound in with that of the staff who care for them. Managers and administrators have the task of enabling those at the bedside to function effectively, and this personal commitment, too, is crucial in the patient's day.

\section{${ }^{1}$ Report by a committee of the Central Health Services Council, The Organisation of the Inpatient's Day. London, HMSO, 1976, price $£ 1 \cdot 35$ \\ Prostatic cancer: policies for progress}

Contentment with things familiar underlies the use of oestrogens as the favourite treatment for carcinoma of the prostate. But discordant notes have been sounded. The Veterans Administration Co-operative Urological Group ${ }^{12}$ has shown that oestrogen, though undoubtedly beneficial by inhibiting the growth of the primary tumour and its metastases, is so at the expense of an increased mortality from cardiovascular disease. When the results of treatment are judged not merely 